\title{
Comment
}

Neuroepidemiology 2010;34:63-64

DOI: $10.1159 / 000264678$

\section{Telephone Interview for Cognitive Status}

Oscar L. Lopez ${ }^{\mathrm{a}, \mathrm{b}}$, Lewis H. Kuller ${ }^{\mathrm{c}}$

Departments of a Neurology and ${ }^{b}$ Psychiatry, University of Pittsburgh School of Medicine, and ${ }^{\mathrm{C}}$ Department of Epidemiology, University of Pittsburgh Graduate School of

Public Health, Pittsburgh, Pa., USA

The paper 'Validation of the Telephone Interview for Cognitive Status-modified in Subjects with Normal Cognition, Mild Cognitive Impairment, or Dementia' from the Mayo Clinic provides a valuable contribution to the evaluation of the Telephone Interview for Cognitive Status-modified (TICS-m) in the identification of mild cognitive impairment (MCI) and dementia [1]. The results strongly suggest that the use of TICS-m is of limited value to ascertain differences between Alzheimer's disease (AD), MCI and normal, at least in older individuals with a very high prevalence of $\mathrm{AD}$ and $\mathrm{MCI}$, but is probably somewhat better in separating dementia and MCI from normal.

The determination of cognitive status in large populations is a major task, and telephone interviews have been proposed as a low-cost alternative to in-person evaluations. They have shown high sensitivity in cohorts with clearly defined dementia [2, 3], but they have had difficulty identifying subjects with MCI and early dementia [4]. The Alzheimer's Disease Cooperative Study found that the TICS-m positive predictive value was $50.9 \%$ in a population of 2,431 subjects screened positive for amnestic MCI [4]. Similarly, the Prevention of Alzheimer's in Society's Elderly found that half of the subjects who had TICS-m scores in the MCI/ early dementia range had the diagnosis of MCI after a clinic evaluation [5]. Therefore, a critical question posed by this paper and similar studies is: what is the utility of the TICS-m in current research related to dementia (especially in its early stages) and $\mathrm{MCI}$ ?

There are two important aspects that are relevant to the determination of cognitive status using telephone interviews. First, the TICS-m may be useful as an initial screening in studies to determine the prevalence of dementia or MCI in a community. At younger ages ( $<75$ years) for example, the prevalence of dementia is relatively low, and therefore a very large sample size is necessary in order to estimate prevalence with reasonable confidence limits. The telephone screening to identify high-risk individuals who could then be further evaluated in order to estimate dementia may be the most cost-effective approach. Nevertheless, the actual determination of the clinical syndrome (i.e. dementia) is based on the secondary clinical evaluation as was originally performed by the National Academy of Sciences Twin Study and the Cache County Study [3]. Unfortunately, even with a fairly high sensitivity and specificity, as suggested by Knopman et al. [1], many socalled mild or early-onset cases of MCI and dementia would likely be missed by this approach because they would have TICS-m scores above the screening cut point for further evaluation. Thus, the estimation of prevalence in the community would then become a function of the cut point used by the TICS- $m$ for the initial screening and the subsequent diagnostic criteria for dementia and MCI. Variations in this two-step approach for identifying dementia cases in the community account, in part, for the variations in the prevalence of dementia across many populations.

Second, the TICS-m can be used in a population study to measure the incidence of dementia over time. However, there is relatively little information to date on the use of the TICS-m to identify incident cases in a longitudinal study. These incident cases are initially likely to be early unless there is a very long time period between examinations. Unless the cut point was set at a very high level, the TICS-m screening may miss many of the early incident cases, similar to the problem in the study of Knopman et al. [1] of discriminating MCI from normal. Recently, in the Ginkgo Evaluation of Memory (GEM) clinical trial, for example, the TICS was used to screen out individuals with a greater likelihood of having dementia, and therefore not being eligible for inclusion into the trial [6]. Such an approach clearly also excludes a certain percentage of individuals who are normal and have scores below the cut point, but this is unlikely to be a problem except perhaps in making it more difficult to recruit in a clinical trial.

There is a substantial possibility of bias with regard to the individuals who are screened above and below the cut point for subsequent evaluation for dementia. Most individuals who have dementia will likely progress to substantial disability, at which point the diagnosis of dementia will become obvious to the family, and data to measure dementia cases can be obtained from the family, from medical records, physicians, and social service agencies with little difficulty. The basic problem is that incident dementia cases may die or develop other serious morbidities which mask the specific dementia diagnosis before they are identified using a screening instrument such as the TICS-m. Thus, the early diagnosed dementia cases may die before they are diagnosed as dementia and, therefore, classified based on their last examination before death as being 'normal'. Consequently, in a longitudinal study of selected risk factors and subsequent incidence of dementia, the risk factors which correlate with both the incidence of dementia and the likelihood of mortality or substantial morbidity and disability will be underestimated in the database.

In a randomized clinical trial, this survival bias prior to diagnosis could be of smaller effect since it theoretically should apply to both the cases in the 'treatment' arm and the controls. Unfor-

\section{KARGER}

Fax +41613061234 E-Mail karger@karger.ch www.karger.com
(อ) 2009 S. Karger AG, Base

$0251-5350 / 10 / 0341-0063 \$ 26.00 / 0$ 
tunately, the situation is not that simple. It is very possible that the 'therapy' has an effect not only on the potential incidence of dementia, but also on the likelihood of survival given the diagnosis of incident dementia. For example, if one was doing a trial of antihypertensive drug therapies for the prevention of dementia, then antihypertensive drug therapy may not only have an effect on reducing the incidence of dementia, but also - by modifying the risk of death or severe disability associated with hypertension (i.e. renal failure, congestive heart failure, stroke, etc.) - increase the life expectancy or the longevity of the incident dementia cases, so that they are more likely to be identified through the screening TICS-m (i.e. as they become more disabled, their TICS-m scores get lower and they reach the cut point for further evaluation). In the control group, on the other hand, that is not receiving the antihypertensive drug therapy, the mortality following the diagnosis of dementia may be much higher than in the treatment arm, and the early cases of dementia may be less likely to be ascertained prior to severe morbidity, i.e. having a disabling stroke, severe renal failure, etc., which makes the diagnosis of dementia prior to the onset of the secondary event very difficult.

The results of the trial may therefore suggest that the antihypertensive drug therapy is actually not very effective, when in truth it might be highly effective but may also result in the decrease in other hypertensive-related morbidity and prolong the duration of the dementia cases, so that they are ascertained in the study based on the screening cut points from the TICS-m or similar instruments. Therefore, one has to be extraordinarily cautious in using a screening cut point in a clinical trial, especially in older individuals with high mortality and comorbidities.

Another basic problem with screening instruments such as the TICS-m is that dementia, or even AD, is clearly not one disease. The TICS-m could provide an estimate of cognitive decline, but probably misses important changes which occur in other various cognitive domains (e.g. visuospatial functions, visual memory) that may be related both to risk factors and especially to treatment in clinical trials. Again, taking the example of antihypertensive drug therapy, it is possible that the therapy preferentially effects frontal lobe changes related to executive function, for example, rather than recent memory. Using the TICS-m as an initial screening device to identify individuals for further testing within the clinical trial may miss important information regarding changes in specific domains of cognition which are important and are related to the specific therapies.

Finally, it is important to consider the role of such screening instruments such as the TICS-m in the modern era of dementia diagnoses, which are increasingly being based on imaging techniques, spinal fluid examinations, etc. Therefore, it might be useful in further evaluations in a population sample to evaluate the specific TICS-m cut points with abnormalities on MRI or PET scanning, etc. For example, do individuals who have similar TICS-m scores or changes in TICS-m scores, but are subsequently classified by further investigation as being dementia, MCI or normal, have a similar or different distribution of pathology based on MRI, PET and so forth?

The fundamental question of using screening instruments in analytical epidemiology such as risk factor longitudinal studies or in clinical trials is whether the potentially reduced cost of using these instruments biases the subsequent results of the study to a degree in which the proper interpretation of the results is no longer feasible or acceptable by the scientific community. The Knopman et al. [1] study has clearly provided more important information regarding the use of the TICS-m in relationship to careful subsequent diagnosis of MCI, dementia and normality. As more large observational studies and clinical trials, especially in the primary prevention of dementia, are underway or planned, the proper methods of measuring outcomes need to be carefully standardized across trials. The critical question of screening cut points - as used in the Knopman et al. [1] study with the TICS-m or a similar instrument with or without subsequent detailed clinical evaluations and imaging techniques - to diagnostic classifications should have a high priority.

The lack of success of primary prevention of dementia and AD as well as the limited ability to identify important risk factors for dementia require very high quality evaluation of dementia cases and their specific characteristics. A penny wise may be a pound foolish.

\section{References}

1 Knopman DS, Roberts RO, Geda YE, Pankratz VS, Christianson THJ, Petersen RC, Rocca WA: Validation of the Telephone Interview for Cognitive Status-modified in subjects with normal cognition, mild cognitive impairment, or dementia. Neuroepidemiology 2010;34:3442 .

2 Brandt J, Spencer M, Folstein M: The Telephone Interview for Cognitive Status. Neuropsychiatry Neuropsychol Behav Neurol 1988;1:111117.

3 Gallo J, Breitner JC: Alzheimer's disease in the NAS-NRC registry of ageing twin veterans, IV. Performance characteristics of a two-stage telephone screening procedure for Alzheimer's disease. Psychol Med 1995;25:1211-1220.

4 Yaari R, Fleisher AS, Gamst AC, Bagwell VP, Thal LJ: Utility of the telephone interview for cognitive enrollment in clinical trials. Alzheimers Dement 2006;2:104-109.

5 Lines CR, McCarrol KA, Lipton RB, Gilbert GA, Prevention of Alzheimer's in Society's Elderly Study Group: Telephone screening for amnestic mild cognitive impairment. Neurology 2003;60:261-266.

6 Dekosky ST, Williamson J, Fitzpatrick A, Kronmal RA, Ives D, Saxton JA; GEM Study Investigators: Ginkgo biloba for primary prevention of dementia: results of the Ginkgo Evaluation of Memory (GEM) study. JAMA 2008;300:2253-2262.

Oscar L. Lopez, MD

3501 Forbes Ave.

Suite 830

Pittsburgh, PA 15213 (USA)

Tel. +1 412246 6970, Fax +1 412246 6873, E-Mail lopezol@upmc.edu 